\title{
On the kinetic systems for simple reacting spheres: modeling and linearized equations
}

\author{
Filipe Carvalho, Jacek Polewczak and Ana Jacinta Soares
}

\begin{abstract}
In this work we present some results on the kinetic theory of chemically reacting gases, concerning the model of simple reacting spheres (SRS) for a gaseous mixture undergoing a chemical reaction of type $A_{1}+A_{2} \rightleftharpoons A_{3}+A_{4}$. Starting from the approach developed in paper [11], we provide properties of the SRS system needed in the mathematical and physical analysis of the model. Our main result in this proceedings provides basic properties of the SRS system linearized around the equilibrium, including the explicit representations of the kernels of the linearized SRS operators.
\end{abstract}

\section{Introduction}

The problem of polyatomic reactive mixtures, within kinetic theory, was first investigated by Prigogine and Xhrouet [13] in 1949. They treated the reactive contributions as perturbations of the elastic terms. This approach is only valid if the reactive cross sections are much smaller than the elastic cross sections. In 1959, Present gave another important contribution to this problem [12]. Although, in some aspects different from the work by Prigogine and Xhrouet, the Present's theory is also based on the assumption that the reactive terms are small perturbations of the elastic terms. Ross and Mazur, in 1961, as well as Shizgal and Karplus, in 1970, see papers $[14,15]$ respectively, used the Chapman-Enskog method in the spatial homogeneous case with the aim of investigating the non-equilibrium effects induced by the chemical reactions and deducing, in particular, the explicit expression of the reaction rate specifying the chemical production of each constituent of the mixture. The works of

Jacek Polewczak

Department of Mathematics, California State University, Northridge, USA, e-mail: jacek. polewczakecsun.edu

Ana Jacinta Soares

Centro de Matemática, Universidade do Minho, Braga, Portugal e-mail: ajsoares@math. uminho.pt

Filipe Carvalho

Centro de Matemática, Universidade do Minho, Braga, Portugal e-mail: filipecarvalho@ esce.ipvc.pt 
Moreau [9], in 1975, and Xystris and Dahler [17], in 1978, used the method of Grad in both spatial homogeneous and inhomogeneous cases with the aim of deducing, again, explicit expressions for the reaction rate.

The kinetic theory of the simple reacting spheres was first proposed by Marron in 1970, see [8], and then developed by Xystris and Dahler in 1978, see [18]. Within this theory, both elastic and reactive collisions are of hard-sphere type. This feature reduces the micro-reversibility principle to a simpler condition.

In 2000, Polewczak proved, in his work [10], the existence of global in time, spatially inhomogeneous, and $L^{1}$-renormalized solution for the model of simple reacting spheres, under the assumption of finite initial mass, momentum and energy. The existence result refers to a four component mixture with a chemical bimolecular reaction in which there was neither mass nor diameters exchange. In this paper we consider a more general situation where the mass and the diameter exchange is allowed. In the dilute-gas limit, this constitutes an interesting kinetic model of chemical reactions that has not yet been studied in detail.

The paper is organized as follows. First, in Section 2, we present the mathematical aspects of the kinetic modeling within the SRS theory. In Sections 3 and 4 we introduce the relevant properties of the collisional operators that are essential to assure the mathematical and physical consistency of the model, and study the tendency of the mixture to approach the equilibrium. In section 5 we define the macroscopic variables and derive the connection of the SRS model to the macroscopic framework in terms of hydrodynamic equations. In Sections 6 and 7 we introduce the linearized SRS system, state its main properties and provide explicit representations for the kernels of the linearized integral operators. Finally, in Section 8 we include a brief discussion about our ongoing research in progress.

\section{Kinetic modeling}

We consider a gas mixture with four constituents, say $A_{1}, \ldots, A_{4}$, with masses $m_{1}, \ldots, m_{4}$ and formation energies $E_{1}, \ldots, E_{4}$, respectively. We restrict our analysis to particles without internal degrees of freedom, which can interact through binary elastic collisions and reactive collisions obeying to the reversible chemical law

$$
A_{1}+A_{2} \rightleftharpoons A_{3}+A_{4}
$$

The mass is conserved during the chemical reaction, so that $m_{1}+m_{2}=m_{3}+m_{4}$. The constituents' indexes are chosen in such a way that the reaction heat, defined by $Q_{R}=E_{3}+E_{4}-E_{1}-E_{2}$, verifies the condition $Q_{R}>0$. This means that the reverse chemical reaction, $A_{3}+A_{4} \rightarrow A_{1}+A_{2}$, is exothermic.

Elastic collisions. An elastic collision between particles $A_{i}$ and $A_{s}$ with velocities $c_{i}$ and $c_{s}$, respectively, results in a change of velocities of both constituents, $\left(c_{i}, c_{s}\right) \rightarrow$ $\left(c_{i}^{\prime}, c_{s}^{\prime}\right)$, with $i, s=1, \ldots, 4$. The conservation laws of linear momentum and kinetic energy of the colliding particles are specified by

$$
m_{i} c_{i}+m_{s} c_{s}=m_{i} c_{i}^{\prime}+m_{s} c_{s}^{\prime}, \quad m_{i} c_{i}^{2}+m_{s} c_{s}^{2}=m_{i} c_{i}^{\prime 2}+m_{s} c_{s}^{\prime 2} .
$$


In our model we consider elastic cross sections of hard-spheres type, given by

$$
\sigma_{i s}^{2}=\frac{1}{4}\left(d_{i}+d_{s}\right)^{2},
$$

where $d_{i}$ and $d_{s}$ denote the diameters of the particle constituents $A_{i}$ and $A_{s}$, respectively. This model of cross sections is one of the most important and frequently used model, mainly due to its simplicity. Conditions (2), together with assumption (3), imply that the elastic post-collisional velocities corresponding to the pre-collisional velocities $c_{i}$ and $c_{s}$ are given by

$$
c_{i}^{\prime}=c_{i}-2 \frac{\mu_{i s}}{m_{i}} \varepsilon\left\langle\varepsilon, c_{i}-c_{s}\right\rangle \quad \text { and } \quad c_{s}^{\prime}=c_{s}+2 \frac{\mu_{i s}}{m_{s}} \varepsilon\left\langle\varepsilon, c_{i}-c_{s}\right\rangle .
$$

Reactive collisions. A reactive collision between particles $A_{i}$ and $A_{j}$ with velocities $c_{i}$ and $c_{j}$, respectively, results in a transition of the reactants $A_{i}$ and $A_{j}$ into products $A_{k}$ and $A_{l}$ and a consequent change of velocities to $c_{k}^{*}$ and $c_{l}^{*}$, with $(i, j, k, l) \in\{(1,2,3,4),(2,1,4,3),(3,4,1,2),(4,3,2,1)\}$. In addition, a reactive collision results in a rearrangement of masses and a redistribution of formation energies. Besides the mass conservation, also the linear momentum and total energy of the colliding particles are preserved, so that the following conditions hold

$$
\begin{aligned}
m_{i} c_{i}+m_{j} c_{j} & =m_{k} c_{k}^{*}+m_{l} c_{l}^{*}, \\
E_{i}+\frac{1}{2} m_{i} c_{i}^{2}+E_{j}+\frac{1}{2} m_{j} c_{j}^{2} & =E_{k}+\frac{1}{2} m_{k} c_{k}^{* 2}+E_{l}+\frac{1}{2} m_{l} c_{l}^{* 2} .
\end{aligned}
$$

In what follows, we use the following notation for the relative velocities of the colliding particles participating in reactive collisions, $\xi_{1}=\xi_{2}=\xi=c_{1}-c_{2}, \xi_{3}=$ $\xi_{4}=\xi^{\prime}=c_{3}-c_{4}$. In the SRS model, reactive collisions are treated as hard-spheres like collisions, with the particularity that a reactive collision between particles $A_{i}$ and $A_{j}$ occurs if the kinetic energy associated with the relative motion along the line of centers exceeds the activation energy. Accordingly, reactive cross sections are assumed in the form

$$
\sigma_{12}^{* 2}=\left\{\begin{array}{ll}
\beta_{12} \sigma_{12}^{2}, & \left\langle\varepsilon, c_{1}-c_{2}\right\rangle \geq \Gamma_{12}, \\
0, & \left\langle\varepsilon, c_{1}-c_{2}\right\rangle<\Gamma_{12},
\end{array} \quad \sigma_{34}^{* 2}= \begin{cases}\beta_{34} \sigma_{34}^{2}, & \left\langle\varepsilon, c_{3}-c_{4}\right\rangle \geq \Gamma_{34}, \\
0, & \left\langle\varepsilon, c_{3}-c_{4}\right\rangle<\Gamma_{34},\end{cases}\right.
$$

for the direct and reverse chemical reaction, respectively. Above, $\beta_{i j}$ is the steric factor for the collision between constituents $A_{i}$ and $A_{j}$, with $0 \leq \beta_{i j} \leq 1$. Moreover, $\Gamma_{i j}$ is a threshold velocity given by $\Gamma_{i j}=\sqrt{2 \gamma_{i} / \mu_{i j}}$, where $\gamma_{i}$ is the activation energy for the constituent $A_{i}$, and $\mu_{i j}=\frac{m_{i} m_{j}}{m_{i}+m_{j}}$ is a reduced mass. The notation $\langle\cdot, \cdot\rangle$ is used for the inner product in $\mathbb{R}^{3}$ and $\varepsilon$ is the unit vector along the line passing through the centers of the spheres at the moment of impact,

$$
\varepsilon \in\left\{\varepsilon \in \mathbb{R}^{3}:|\varepsilon|=1 \wedge\left\langle\varepsilon, c_{i}-c_{j}\right\rangle \geq 0\right\} \equiv \mathbb{S}_{+}^{2} .
$$


Notice that, for the chemical reaction defined in Eq. (1), the activation energies verify the conditions $\gamma_{2}=\gamma_{1}, \gamma_{3}=\gamma_{1}-Q_{R}, \gamma_{4}=\gamma_{3}$ and the steric factors are such that $\beta_{i j}=0$, for $(i, j) \notin\{(1,2),(2,1),(3,4),(4,3)\}$. Furthermore, since the reaction heat $Q_{R}$ is positive, we must have $\gamma_{1}>Q_{R}$.

In our SRS model, the post-collisional velocities for the direct chemical reaction are given by

$$
\begin{aligned}
& c_{3}^{*}=\frac{1}{M}\left[m_{1} c_{1}+m_{2} c_{2}+m_{4} \sqrt{\frac{\mu_{12}}{\mu_{34}}}\left\{\xi-\varepsilon\langle\varepsilon, \xi\rangle+\varepsilon \alpha^{-}\right\}\right], \\
& c_{4}^{*}=\frac{1}{M}\left[m_{1} c_{1}+m_{2} c_{2}-m_{3} \sqrt{\frac{\mu_{12}}{\mu_{34}}}\left\{\xi-\varepsilon\langle\varepsilon, \xi\rangle+\varepsilon \alpha^{-}\right\}\right],
\end{aligned}
$$

whereas, the post-collisional velocities for the reverse chemical reaction are

$$
\begin{aligned}
& c_{1}^{*}=\frac{1}{M}\left[m_{3} c_{3}+m_{4} c_{4}+m_{2} \sqrt{\frac{\mu_{34}}{\mu_{12}}}\left\{\xi^{\prime}-\varepsilon\left\langle\varepsilon, \xi^{\prime}\right\rangle+\varepsilon \alpha^{+}\right\}\right] \\
& c_{2}^{*}=\frac{1}{M}\left[m_{3} c_{3}+m_{4} c_{4}-m_{1} \sqrt{\frac{\mu_{34}}{\mu_{12}}}\left\{\xi^{\prime}-\varepsilon\left\langle\varepsilon, \xi^{\prime}\right\rangle+\varepsilon \alpha^{+}\right\}\right] .
\end{aligned}
$$

where $\alpha^{-}=\sqrt{(\langle\varepsilon, \xi\rangle)^{2}-2 Q_{R} / \mu_{12}}$ and $\alpha^{+}=\sqrt{\left(\left\langle\varepsilon, \xi^{\prime}\right\rangle\right)^{2}+2 Q_{R} / \mu_{34}}$. Velocities $c_{i}$, $c_{j}, c_{k}^{*}, c_{l}^{*}$, with $(i, j, k, l) \in\{(1,2,3,4),(2,1,4,3),(3,4,1,2),(4,3,2,1)\}$ and $c_{k}^{*}, c_{l}^{*}$ given by expressions (8-11), verify the conservation laws expressed in Eqs. (5-6).

Kinetic equations. In the absence of external forces, the kinetic equations, describing the time-space evolution of the one-particle distribution functions $f_{i}\left(x, c_{i}, t\right)$, $i=1, \ldots, 4$, can be written in the form

$$
\frac{\partial f_{i}}{\partial t}+\sum_{l=1}^{3} c_{l}^{i} \frac{\partial f_{i}}{\partial x_{l}}=\mathscr{Q}_{i}^{E}+\mathscr{Q}_{i}^{R}, \quad i=1, \ldots, 4,
$$

where $\mathscr{Q}_{i}^{E}$ and $\mathscr{Q}_{i}^{R}$ represent the elastic and reactive collisional operators. Following paper [11], the collisional operators have the form

$$
\begin{aligned}
\mathscr{Q}_{i}^{E}= & \sum_{s=1}^{4}\left\{\sigma_{i s}^{2} \int_{\mathbb{R}^{3}} \int_{\mathbb{S}_{+}^{2}}\left[f_{i}^{\prime} f_{s}^{\prime}-f_{i} f_{s}\right]\left\langle\varepsilon, c_{i}-c_{s}\right\rangle d \varepsilon d c_{s}\right\} \\
& -\beta_{i j} \sigma_{i j}^{2} \int_{\mathbb{R}^{3}} \int_{\mathbb{S}_{+}^{2}}\left[f_{i}^{\prime} f_{j}^{\prime}-f_{i} f_{j}\right] \Theta\left(\left\langle\varepsilon, \xi_{i}\right\rangle-\Gamma_{i j}\right)\left\langle\varepsilon, \xi_{i}\right\rangle d \varepsilon d c_{j},
\end{aligned}
$$

where $\Theta$ is the Heaviside step function and $(i, j) \in\{(1,2),(2,1),(3,4),(4,3)\}$, and

$$
\mathscr{Q}_{i}^{R}=\beta_{i j} \sigma_{i j}^{2} \int_{\mathbb{R}^{3}} \int_{\mathbb{S}_{+}^{2}}\left[\left(\frac{\mu_{i j}}{\mu_{k l}}\right)^{2} f_{k}^{*} f_{l}^{*}-f_{i} f_{j}\right] \Theta\left(\left\langle\varepsilon, \xi_{i}\right\rangle-\Gamma_{i j}\right)\left\langle\varepsilon, \xi_{i}\right\rangle d \varepsilon d c_{j},
$$


where $(i, j, k, l) \in\{(1,2,3,4),(2,1,4,3),(3,4,1,2),(4,3,2,1)\}$, and $f_{k}^{*}=f_{k}\left(x, c_{k}^{*}, t\right)$, $f_{l}^{*}=f_{l}\left(x, c_{l}^{*}, t\right)$. As explained in papers $[11,18]$, the second term in the expression of $\mathscr{Q}_{i}^{E}$ is a correction term for the occurrence of reactive collisions and prevent a double counting of the contributions in the collisional operators. In fact, those encounters between $A_{i}$ and $A_{j}$ particles which are sufficiently energetic in the sense that $\left\langle\varepsilon, \xi_{i}\right\rangle \geq \Gamma_{i j}$ result in chemical reaction and should not be counted as elastic encounters.

\section{Properties of the collisional operators}

The consistency of the model is assured when the collisional operators have some important properties. We begin with the following fundamental results, concerning the elastic and reactive operators.

Proposition 1 If we assume that $\beta_{i j}=\beta_{j i}$ then, for $\psi_{i}$ measurable on $\mathbb{R}^{3}$ and $f_{i} \in$ $C_{0}\left(\mathbb{R}^{3}\right), i=1, \ldots, 4$, we have

$$
\begin{aligned}
\int_{\mathbb{R}^{3}} \psi_{i} \mathscr{Q}_{i}^{E} d c_{i}= & \frac{1}{4} \sum_{s=1}^{4}\left\{\sigma_{i s}^{2} \int_{\mathbb{R}^{3}} \int_{\mathbb{R}^{3}} \int_{\mathbb{S}_{+}^{2}}\left[\psi_{i}+\psi_{s}-\psi_{i}^{\prime}-\psi_{s}^{\prime}\right]\left[f_{i}^{\prime} f_{s}^{\prime}-f_{i} f_{s}\right]\left\langle\varepsilon, c_{i}-c_{s}\right\rangle d \varepsilon d c_{s} d c_{i}\right\} \\
& -\frac{1}{4} \beta_{i j} \sigma_{i j}^{2} \int_{\mathbb{R}^{3}} \int_{\mathbb{R}^{3}} \int_{\mathbb{S}_{+}^{2}}\left[\psi_{i}+\psi_{j}-\psi_{i}^{\prime}-\psi_{j}^{\prime}\right]\left[f_{i}^{\prime} f_{j}^{\prime}-f_{i} f_{j}\right] \\
& \times \Theta\left(\left\langle\varepsilon, \xi_{i}\right\rangle-\Gamma_{i j}\right)\left\langle\varepsilon, \xi_{i}\right\rangle d \varepsilon d c_{j} d c_{i} .
\end{aligned}
$$

Proposition 2 If $\beta_{i j}=\beta_{j i}$ and $\beta_{12} \sigma_{12}^{2}=\beta_{34} \sigma_{34}^{2}$, then we have

$$
\begin{aligned}
\sum_{i=1}^{4} \int_{\mathbb{R}^{3}} \psi_{i} \mathscr{Q}_{i}^{R} d c_{i}= & \beta_{12} \sigma_{12}^{2} \int_{\mathbb{R}^{3}} \int_{\mathbb{R}^{3}} \int_{\mathbb{S}_{+}^{2}}\left[\psi_{1}+\psi_{2}-\psi_{3}^{*}-\psi_{4}^{*}\right] \\
& \times\left[\left(\frac{\mu_{12}}{\mu_{34}}\right)^{2} f_{3}^{*} f_{4}^{*}-f_{1} f_{2}\right] \Theta\left(\langle\varepsilon, \xi\rangle-\Gamma_{12}\right)\langle\varepsilon, \xi\rangle d \varepsilon d c_{2} d c_{1} \\
= & \beta_{34} \sigma_{34}^{2} \int_{\mathbb{R}^{3}} \int_{\mathbb{R}^{3}} \int_{\mathbb{S}_{+}^{2}}\left[\psi_{3}+\psi_{4}-\psi_{1}^{*}-\psi_{2}^{*}\right] \\
& \times\left[\left(\frac{\mu_{34}}{\mu_{12}}\right)^{2} f_{1}^{*} f_{2}^{*}-f_{3} f_{4}\right] \Theta\left(\left\langle\varepsilon, \xi^{\prime}\right\rangle-\Gamma_{34}\right)\left\langle\varepsilon, \xi^{\prime}\right\rangle d \varepsilon d c_{4} d c_{3} .
\end{aligned}
$$

Propositions 1 and 2 can be proven considering the symmetry properties of the collisional operators, and constitute the basis of the proof of the following results.

Proposition 3 The elastic collisional operators are such that

$$
\int_{\mathbb{R}^{3}} \mathscr{Q}_{i}^{E} d c_{i}=0, \quad i=1, \ldots, 4 .
$$

Proposition 3 states that elastic encounters do not change the number of particles of each constituent. 
Proposition 4 The reactive collisional operators satisfy the following properties

$$
\int_{\mathbb{R}^{3}} \mathscr{Q}_{1}^{R} d c_{1}=\int_{\mathbb{R}^{3}} \mathscr{Q}_{2}^{R} d c_{2}=-\int_{\mathbb{R}^{3}} \mathscr{Q}_{3}^{R} d c_{3}=-\int_{\mathbb{R}^{3}} \mathscr{Q}_{4}^{R} d c_{4} .
$$

Proposition 4 states that the variation of the number of particles of constituent $A_{1}$ is the same as that of constituent $A_{2}$ and symmetric to the variation of constituents $A_{3}$ and $A_{4}$. It assures the correct chemical exchange rates of the constituents predicted by the reaction mechanism.

Definition 1 A function $\psi=\left(\psi_{1}, \psi_{2}, \psi_{3}, \psi_{4}\right)$ is a collisional invariant in the velocity space, for the SRS model, if

$$
\sum_{i=1}^{4} \int_{\mathbb{R}^{3}} \psi_{i}\left(\mathscr{Q}_{i}^{E}+\mathscr{Q}_{i}^{R}\right) d c_{i}=0 .
$$

The following Proposition 5 presents the collisional invariants of model and establishes the consistency of the model from the physical point of view.

Proposition 5 Functions $\psi=(1,0,1,0), \psi=(1,0,0,1), \psi=(0,1,1,0)$, and functions $\psi=\left(\psi_{1}, \psi_{2}, \psi_{3}, \psi_{4}\right)$ defined by $\psi_{i}=m_{i} c_{1}^{i}, \psi_{i}=m_{i} c_{2}^{i}, \psi_{i}=m_{i} c_{3}^{i}$ and $\psi_{i}=$ $E_{i}+\frac{1}{2} c_{i}^{2} m_{i}$ are collisional invariants.

The first three invariants assure the conservation of the partial number density of a pair of constituents, one reactant and one product of the chemical reaction, namely $A_{1}$ and $A_{3}, A_{1}$ and $A_{4}$, and $A_{2}$ and $A_{3}$, respectively. They also assure the conservation of the total number density of the reactive mixture. The next three invariants assure the conservation of the linear momentum components of the mixture, whereas the last invariant assures the conservation of the total energy of the reactive mixture.

\section{Equilibrium distributions and the Boltzmann H-theorem}

When the gas reaches the equilibrium the elastic and reactive collisions do not stop, they become balanced. This means that, when the mixture is at equilibrium conditions, the collisional process does not modify the one-particle distibutions $f_{i}$. In particular, the number of particles that enter a volume element in the phase space per unit time is the same as the number of particles that leave the volume element in the phase space per unit time.

Definition 2 The gas mixture is in thermodynamical equilibrium when the elastic and reactive collisional operators are such that

$$
\mathscr{Q}_{i}^{E}+\mathscr{Q}_{i}^{R}=0, \quad i=1, \ldots, 4 .
$$

In particular, in our model, condition (18) implies the vanishing of the elastic collisional operators,

$$
\mathscr{Q}_{i}^{E}=0, \quad i=1, \ldots, 4 .
$$


Condition (19), in absence of reactive terms, is usually called a state of mechanical equilibrium.

The following Proposition 6 is well known in the case on one-single component gas and a formal proof can be found in many books, see for example [3].

Proposition 6 If all constituents are at the same temperature, the only distribution function that assures the mechanical equilibrium is the Maxwellian distribution

$$
f_{i}^{M}\left(x, c_{i}, t\right)=n_{i}\left(\frac{m_{i}}{2 \pi k T}\right)^{\frac{3}{2}} \exp \left[-\frac{m_{i}\left(c_{i}-v\right)^{2}}{2 k T}\right], \quad i=1, \ldots, 4,
$$

where $n_{i}, T$ and $v$ are the number density of constituent $A_{i}$, temperature and mean velocity of the whole mixture, respectively, and $k$ is the Boltzmann constant.

The above Maxwellian distributions (20) do not assure, in general, the vanishing of the reactive collisional operators and thus do not define a state of thermodynamical equilibrium for the reactive mixture.

Proposition 7 If all constituents are at the same temperature, the only distribution function that assures the thermodynamical equilibrium is the thermodynamical Maxwellian distribution given by

$$
M_{i}\left(x, c_{i}, t\right)=n_{i}\left(\frac{m_{i}}{2 \pi k T}\right)^{3 / 2} \exp \left[-\frac{m_{i}\left(c_{i}-v\right)^{2}}{2 k T}\right], \quad i=1, \ldots, 4,
$$

with the number densities $n_{i}$ constrained to the condition

$$
n_{1} n_{2}=n_{3} n_{4} \sqrt{\frac{\mu_{12}}{\mu_{34}}} \exp \left(\frac{Q_{R}}{k T}\right) .
$$

Equation (22) represents the law of mass action for the SRS model.

The important physical feature of trend to equilibrium is now presented in the specific case of spatial domain $\Omega=\mathbb{R}^{3}$, proving the existence of an $\mathrm{H}$-function (Liapunov functional) of the SRS system (12), (13) and (14).

Proposition 8 ( $\mathscr{H}$-theorem) If the steric factors $\beta_{i j}$ and cross sections $\sigma_{i j}$ are such that $\beta_{i j}=\beta_{j i}$ and $\beta_{12} \sigma_{12}^{2}=\beta_{34} \sigma_{34}^{2}$, the convex function $\mathrm{H}(t)$, defined by

$$
\mathrm{H}(t)=\sum_{i=1}^{4} \int_{\Omega} \int_{\mathbb{R}^{3}} f_{i} \log \left(\frac{f_{i}}{\mu_{i j}}\right) d c_{i} d x,
$$

where $(i, j) \in\{(1,2),(2,1),(3,4),(4,3)\}$ and $f_{i} \in L^{1}\left(\Omega \times \mathbb{R}^{3}\right)$ for all $i=1, \ldots, 4$, is an $H$-function (Liapunov functional) for the SRS system (12), (13) and (14), that is

$$
\begin{aligned}
& \frac{d \mathrm{H}}{d t}(t) \leq 0 \quad \text { for all } \quad t \geq 0, \quad \text { and } \\
& \frac{d \mathrm{H}}{d t}(t)=0 \quad \text { if and only if } \quad f_{i}=M_{i} \text { for all } i=1, \ldots, 4 .
\end{aligned}
$$


In the case of a spatial homogeneous evolution, the domain $\Omega$ is irrelevant for the

behavior of the corresponding $H$-function, $\mathscr{H}(t)=\sum_{i=1}^{4} \int_{\mathbb{R}^{3}} f_{i} \log \left(\frac{f_{i}}{\mu_{i j}}\right) d c_{i}$. In the general case considered in Proposition 8, there exists a limited range of known situations for which the result is still valid. Some of them correspond to consider $\Omega$ as a box with boundary conditions of periodic type or boundary conditions of specular reflection at the walls, see for instance Refs. [3, 16].

The result expressed in Proposition 8 states that the reactive mixture evolves to a thermodynamical equilibrium state. In particular, in the proof of this proposition one shows that both elastic and reactive collisions contribute, independently, to this tendency to equilibrium. The spatial homogeneous version of an $\mathscr{H}$-theorem, similar to Proposition 8, is proven in paper [7], for a kinetic model for a quaternary reactive mixture undergoing a reversible bimolecular reaction. In comparison to the SRS model studied in our paper, the kinetic model considered in paper [7] has two major differences. First, the reactive cross sections of paper [7] follow the line-ofcenters model, in contrast to those considered in our paper which are of hard-sphere type. Second, contrarily to the SRS system, the model of paper [7] does not consider any correction term in the collisional operators for preventing a double counting of the contributions. See the explanations at the end of Section 2.

In paper [7], the authors use their $\mathscr{H}$-theorem to prove the strong convergence in $L^{1}$-sense of the solution of their kinetic system to a Maxwellian distribution of thermodynamical equilibrium, under the assumption of uniformly boundedness and equicontinuity of the distribution functions for the spatial homogeneous case. Thus, in our opinion, Proposition 8 should constitute a central result in the convergence analysis of the solution of the kinetic equations (12) to a thermodynamical Maxwellian distribution.

\section{Macroscopic framework}

It is well known that the Boltzmann equation constitutes a fundamental model in the kinetic theory of gases, that describes the dynamics of the gas particles. At the same time, in the hydrodynamic limit, it leads to a description in terms of physically meaningful macroscopic quantities and related balance equations. The same happens with the SRS model studied in this paper. The mathematical and physical properties of the collisional operators, stating the consistency of the SRS system, are fundamental for the validity of the model as well as for the passage to the hydrodynamic limit. From a formal point of view, the connection between the microscopic variables and the macroscopic framework is based on the idea that all measurable macroscopic quantities can be expressed in terms of microscopic averages of the distribution functions. We now define the macroscopic quantities of the SRS model and provide the evolution equations for the most relevant macroscopic quantities.

Macroscopic variables. As usual, we define the macroscopic variables as suitable moments of the distribution functions $f_{i}$. We use the index $i$ for those quantities 
associated to the constituent $A_{i}, i=1, \ldots, 4$, and denote with plain symbols the macroscopic variables referred to the whole mixture. Moreover, indexes $l$ and $j$ are used to represent spatial components of vectorial quantities in $\mathbb{R}^{3}$.

Number density

$$
n_{i}=\int_{\mathbb{R}^{3}} f_{i} d \mathbf{c}_{i} \text { and } n=\sum_{i=1}^{4} n_{i}
$$

Mass density

$$
\rho_{i}=\int_{\mathbb{R}^{3}} m_{i} f_{i} d \mathbf{c}_{i} \quad \text { and } \quad \rho=\sum_{i=1}^{4} \rho_{i}
$$

Momentum density

$$
\rho_{i} v_{i}=\int_{\mathbb{R}^{3}} m_{i} c_{i} f_{i} d \mathbf{c}_{i} \quad \text { and } \quad \rho v=\sum_{i=1}^{4} \rho_{i} v_{i}
$$

Diffusion velocity

$$
u_{i}=\frac{1}{\rho_{i}} \int_{\mathbb{R}^{3}} m_{i} \zeta_{i} f_{i} d \mathbf{c}_{i}
$$

Pressure

$$
p_{i}=\frac{1}{3} \int_{\mathbb{R}^{3}} m_{i} \zeta_{i}^{2} f_{i} d \mathbf{c}_{i} \text { and } p=\sum_{i=1}^{4} p_{i}
$$

Pressure tensor components

Temperature

$$
p_{l j}^{i}=\int_{\mathbb{R}^{3}} m_{i} \zeta_{l}^{i} \zeta_{j}^{i} f_{i} d \mathbf{c}_{i} \quad \text { and } \quad p_{l j}=\sum_{i=1}^{4} p_{l j}^{i}
$$

Heat flux components

$$
\begin{aligned}
& T_{i}=\frac{p_{i}}{n_{i} k} \quad \text { and } \quad T=\sum_{i=1}^{4} \frac{n_{i}}{n} T_{i}=\frac{p}{n k} \\
& q_{l}^{i}=\int_{\mathbb{R}^{3}} \frac{1}{2} m_{i} \zeta_{i}^{2} \zeta_{l}^{i} f_{i} d c_{i} \quad \text { and } \quad q_{l}=\sum_{i=1}^{4}\left(q_{l}^{i}+n_{i} E_{i} u_{l}^{i}\right)
\end{aligned}
$$

where $\zeta_{i}=c_{i}-v$ is the peculiar velocity, and $\zeta_{l}^{i}$ or $\zeta_{j}^{i}$ represent its spatial components. Moreover, the term $n_{i} E_{i} u_{l}^{i}$ in the definition of $q_{l}$ refers to the formation energy transfer of the constituent $A_{i}$ due to diffusion.

Balance equations. By multiplying the SRS equations (12) by suitable functions $\psi_{i}$, and then integrating over $c_{i} \in \mathbb{R}^{3}$, one can derive the balance equations for each constituent $A_{i}$. Omitting here the details, the balance equations for the number density, linear momentum components and total energy of each constituent $A_{i}, i=1, \ldots, 4$, have the form

$$
\begin{gathered}
\frac{\partial n_{i}}{\partial t}+\sum_{l=1}^{3} \frac{\partial}{\partial x_{l}}\left(n_{i} u_{l}^{i}+n_{i} v_{l}\right)=\int_{\mathbb{R}^{3}}\left(\mathscr{Q}_{i}^{E}+\mathscr{Q}_{i}^{R}\right) d \mathbf{c}_{i} \\
\frac{\partial}{\partial t}\left(\rho_{i} v_{l}^{i}\right)+\sum_{r=1}^{3} \frac{\partial}{\partial x_{r}}\left(p_{l r}^{i}+\rho_{i} u_{l}^{i} v_{r}+\rho_{i} u_{r}^{i} v_{l}+\rho_{i}\right)=\int_{\mathbb{R}^{3}} m_{i} c_{l}^{i}\left(\mathscr{Q}_{i}^{E}+\mathscr{Q}_{i}^{R}\right) d \mathbf{c}_{i} \\
\frac{\partial}{\partial t}\left[\frac{3}{2} p_{i}+n_{i} E_{i}+\rho_{i} u_{l}^{i} v_{l}+\frac{1}{2} \rho_{i} v^{2}\right]+\sum_{l=1}^{3} \frac{\partial}{\partial x_{l}}\left[q_{l}^{i}+p_{l r}^{i} v_{r}+n_{i} E_{i} u_{l}^{i}+\frac{1}{2} \rho_{i} u_{l}^{i} v^{2}\right. \\
\left.+\left(\frac{3}{2} p_{i}+n_{i} E_{i}+\rho_{i} u_{l}^{i} v_{l}+\frac{1}{2} \rho_{i} v^{2}\right) v_{i}\right] \\
=\int_{\mathbb{R}^{3}}\left(\frac{1}{2} m_{i} c_{i}^{2}+E_{i}\right)\left(\mathscr{Q}_{i}^{E}+\mathscr{Q}_{i}^{R}\right) d \mathbf{c}_{i} .
\end{gathered}
$$


In particular, Eq. (24) constitutes the reaction rate equation of the SRS system and specifies the production rate of each constituent of the gas mixture.

Conservation laws. The conservation equations for partial number densities are obtained from the balance equations (24) by summing over one reactant $(i=1,2)$ and one product $(i=3,4)$ of the chemical reaction. Moreover, the conservation equations for mass density, linear momentum components and total energy of the whole mixture are obtained from the balance equations (24-26) by summing over all constituents. They can be written in the form

$$
\begin{aligned}
& \frac{\partial}{\partial t}\left(n_{i}+n_{j}\right)+\sum_{l=1}^{3} \frac{\partial}{\partial x_{l}}\left[n_{i} u_{l}^{i}+n_{j} u_{l}^{j}+\left(n_{i}+n_{j}\right) v_{l}^{i}\right]=0, \quad i=1,2, j=3,4, \\
& \frac{\partial \rho}{\partial t}+\sum_{l=1}^{3} \frac{\partial}{\partial x_{l}}\left(\rho v_{l}\right)=0, \\
& \frac{\partial}{\partial t}\left(\rho v_{l}\right)+\sum_{k=1}^{3} \frac{\partial}{\partial x_{k}}\left(p_{l k}+\rho v_{l} v_{k}\right)=0, \quad l=1,2,3, \\
& \frac{\partial}{\partial t}\left(\frac{3}{2} n k T+\sum_{i=1}^{4} n_{i} E_{i}+\frac{1}{2} \rho v^{2}\right)+ \sum_{l=1}^{3} \frac{\partial}{\partial x_{l}}\left[q_{l}+\sum_{l=1}^{3} p_{l k} v_{k}\right. \\
&\left.+\left(\frac{3}{2} n k T+\sum_{i=1}^{4} n_{i} E_{i}+\frac{1}{2} \rho v^{2}\right) v_{l}\right]=0 .
\end{aligned}
$$

\section{Linearized SRS system}

The linearized formulation of the SRS system around thermodynamical equilibrium arises as a simplification of the full system, which is valid when the reactive mixture is close to the thermodynamical equilibrium. In this section we construct the linearized SRS kinetic system and state its fundamental properties.

Linearized SRS system. To obtain the linearized equations, first the distribution function $f_{i}$ is expanded around the thermodynamical Maxwellian distribution $M_{i}$ with zero drifting velocity $(v=0)$, in the form

$$
f_{i}\left(x, c_{i}, t\right)=M_{i}\left(x, c_{i}, t\right)\left[1+h_{i}\left(x, c_{i}, t\right)\right], \quad i=1, \ldots, 4,
$$

where $h_{i}$ represents the deviation of the distribution function from the equilibrium. Then, expansions (31) are inserted into the SRS system (12-14) and the conservation laws (2) and (5-6) associated to elastic and reactive collisions, respectively, are used, together with the law of mass action (22). In the sequel we introduce the notation $\underline{w}=\left(w_{1}, w_{2}, w_{3}, w_{4}\right)^{T}$.

Proposition 9 If we neglect quadratic and higher order terms in the deviations $h_{i}$, the linearized SRS system takes the form 
On the kinetic systems for simple reacting spheres: modeling and linearized equations

$$
\frac{\partial h_{i}}{\partial t}+\sum_{l=1}^{3} c_{l}^{i} \frac{\partial h_{i}}{\partial x_{l}}=\mathscr{L}_{i}^{E}(\underline{h})+\mathscr{L}_{i}^{R}(\underline{h}) \equiv \mathscr{L}_{i}(\underline{h}), \quad i=1, \ldots, 4,
$$

with

$$
\begin{aligned}
\mathscr{L}_{i}^{E}(\underline{h})= & \sum_{s=1}^{4} \sigma_{i s}^{2} \int_{\mathbb{R}^{3}} \int_{\mathbb{S}_{+}^{2}} M_{s}\left[h_{i}^{\prime}+h_{s}^{\prime}-h_{i}-h_{s}\right]\left\langle\varepsilon, c_{i}-c_{s}\right\rangle d \varepsilon d c_{s} \\
& -\beta_{i j} \sigma_{i j}^{2} \int_{\mathbb{R}^{3}} \int_{\mathbb{S}_{+}^{2}} M_{j}\left[h_{i}^{\prime}+h_{j}^{\prime}-h_{i}-h_{j}\right] \Theta\left(\left\langle\varepsilon, \xi_{i}\right\rangle-\Gamma_{i j}\right)\left\langle\varepsilon, \xi_{i}\right\rangle d \varepsilon d c_{j},
\end{aligned}
$$

for $(i, j) \in\{(1,2),(2,1),(3,4),(4,3)\}$, and

$$
\mathscr{L}_{i}^{R}(\underline{h})=\beta_{i j} \sigma_{i j}^{2} \int_{\mathbb{R}^{3}} \int_{\mathbb{S}_{+}^{2}} M_{j}\left[h_{k}^{*}+h_{l}^{*}-h_{i}-h_{j}\right] \Theta\left(\left\langle\varepsilon, \xi_{i}\right\rangle-\Gamma_{i j}\right)\left\langle\varepsilon, \xi_{i}\right\rangle d \varepsilon d c_{j},
$$

for $(i, j, k, l) \in\{(1,2,3,4),(2,1,4,3),(3,4,1,2),(4,3,2,1)\}$.

Properties of the linearized SRS system. Some important mathematical properties of the linearized SRS system (32-34) will be presented in the sequel. In order to easily compare our results with previous ones existing in literature for inert gases, we consider the following weighted distribution function and weighted operator,

$$
\widehat{f}_{i}=M_{i}^{1 / 2} f_{i} \quad \text { and } \quad \widehat{\mathscr{L}_{i}}(\underline{\hat{h}})=M_{i}^{1 / 2} \mathscr{L}_{i}(\underline{h}), \quad i=1, \ldots, 4 .
$$

We can easily verify that $h_{i}$ defines a solution of the linearized SRS system (32-34) if and only if $\hat{h}_{i}$ defines a solution of the following weighted linearized system

$$
\frac{\partial \hat{h}_{i}}{\partial t}+\sum_{l=1}^{3} c_{l}^{i} \frac{\partial \hat{h}_{i}}{\partial x_{l}}=\widehat{\mathscr{L}_{i}}(\underline{\hat{h}}), \quad i=1, \ldots, 4
$$

where the weighted linearized operator $\widehat{\mathscr{L}_{i}}(\underline{\hat{h}})$ can be split in its elastic and reactive parts, given by

$$
\widehat{\mathscr{L}}_{i}^{E}(\underline{\hat{h}})=M_{i}^{1 / 2} \mathscr{L}_{i}^{E}(h) \quad \text { and } \quad \widehat{\mathscr{L}}_{i}^{R}(\underline{\hat{h}})=M_{i}^{1 / 2} \mathscr{L}_{i}^{R}(h) .
$$

Moreover, we introduce the space $Y=L^{2}\left(\mathbb{R}^{3} \times \mathbb{R}^{3}\right)$ and consider the Maxwellian weighted velocity $L^{2}$-space, $Y^{4}$, endowed with the inner product defined by

$$
\langle\underline{F}, \underline{G}\rangle=\sum_{i=1}^{4} \int_{\mathbb{R}^{3}} F_{i}\left(c_{i}\right) G_{i}\left(c_{i}\right) d c_{i} .
$$

The weighted linearized collisional operator satisfies the following property.

Proposition 10 If the steric factors $\beta_{i j}$ and cross sections $\sigma_{i j}$ are such that $\beta_{i j}=\beta_{j i}$ and $\beta_{12} \sigma_{12}^{2}=\beta_{34} \sigma_{34}^{2}$, the weighted linearized collisional operator $\widehat{\mathscr{L}}$ is symmetric and non-positive semi-definite, that is 
(a) $\langle\underline{\hat{g}}, \widehat{\mathscr{L}}(\underline{\hat{h}})\rangle=\langle\underline{\hat{h}}, \widehat{\mathscr{L}}(\underline{\hat{g}})\rangle$, for all $\underline{g}, \underline{h} \in Y^{4}$;

(b) $\langle\underline{\hat{h}}, \widehat{\mathscr{L}}(\underline{\hat{h}})\rangle \leq 0$, for all $\underline{h} \in Y^{4}$, and $\langle\underline{\hat{h}}, \widehat{\mathscr{L}}(\underline{\hat{h}})\rangle=0$ if and only if $\underline{h}$ is a collisional invariant.

\section{Kernels of the linearized integral operators}

For a one component inert gas, the explicit expression of the kernel of the linearized collisional operator, as well as the techniques used to compute the kernel, are detailed in many works, in particular in paper [4]. However, this is not the case for a reactive gas mixture. In fact, the computations for the case of a reactive gas mixture are long and very technical. Concerning, in particular, the SRS system, we were able to obtain the explicit representation of the kernels of the linearized elastic and reactive operators, in the general case of arbitrary molecular masses. The computations have been done by the first author of the present work and are part of his $\mathrm{PhD}$ thesis, see [1]. See also Ref. [2] for the details about the computations of the kernels.

Kernels of the linearized elastic operators. The operator $\widehat{\mathscr{L}}_{i}^{E}(\underline{\hat{h}})$ can be split into several contributions as follows

$$
\widehat{\mathscr{L}}_{i}^{E}(\underline{\hat{h}})=-v_{i} \hat{h}_{i}-Q_{i}^{(1)}(\underline{\hat{h}})+Q_{i}^{(2)}(\underline{\hat{h}})+Q_{i}^{(3)}(\underline{\hat{h}})
$$

where

$$
\begin{aligned}
& v_{i} \hat{h_{i}}=\hat{h_{i}} \sum_{s=1}^{4} \sigma_{i s}^{2} \int_{\mathbb{R}^{3}} \int_{\mathbb{S}_{+}^{2}} M_{s}\left\langle\varepsilon, c_{i}-c_{s}\right\rangle d \varepsilon d c_{s}, \\
& Q_{i}^{(1)}(\underline{\hat{h}})=\sum_{s=1}^{4} \sigma_{i s}^{2} \int_{\mathbb{R}^{3}} \int_{\mathbb{S}_{+}^{2}} M_{i}^{1 / 2} M_{s}^{1 / 2} \hat{h}_{s}\left\langle\varepsilon, c_{i}-c_{s}\right\rangle d \varepsilon d c_{s}, \\
& Q_{i}^{(2)}(\underline{\hat{h}})=\sum_{s=1}^{4} \sigma_{i s}^{2} \int_{\mathbb{R}^{3}} \int_{\mathbb{S}_{+}^{2}} M_{i}^{1 / 2} M_{s} M_{i}^{\prime-1 / 2} \hat{h}_{i}^{\prime}\left\langle\varepsilon, c_{i}-c_{s}\right\rangle d \varepsilon d c_{s}, \\
& Q_{i}^{(3)}(\underline{\hat{h}})=\sum_{s=1}^{4} \sigma_{i s}^{2} \int_{\mathbb{R}^{3}} \int_{\mathbb{S}_{+}^{2}} M_{i}^{1 / 2} M_{s} M_{s}^{\prime-1 / 2} \hat{h}_{s}^{\prime}\left\langle\varepsilon, c_{i}-c_{s}\right\rangle d \varepsilon d c_{s} .
\end{aligned}
$$

The multiplication operator $v_{i}$ defined in (40) can be identified as a mean collision frequency. Concerning the integral operators (41-43), the full representation of the kernels, in the general case of arbitrary molecular masses, is omitted here due to space limitations. In fact, some of the expressions are very long. We only include the expressions of the kernels in the particular case of a reactive mixture with equal molecular masses. They are given by the following expressions, for $i=1,2,3,4$,

$$
\begin{aligned}
& N\left(Q_{i}^{(1)}\right)(u, w)=\pi \sigma_{i s}^{2}\|u-w\| \sqrt{n_{i} n_{s}}\left(\frac{m}{2 \pi k T}\right)^{3 / 2} \exp \left(-\frac{m\left(u^{2}+w^{2}\right)}{4 k T}\right), \\
& N\left(Q_{i}^{(2)}\right)(u, w)=\sigma_{i s}^{2} n_{s}\left(\frac{m}{2 \pi k T}\right)^{1 / 2} \frac{1}{\|u-w\|}
\end{aligned}
$$




$$
\begin{aligned}
& \times \exp \left[-\frac{m}{8 k T} \frac{\left(u^{2}-w^{2}\right)^{2}}{\|u-w\|^{2}}-\frac{m}{8 k T}(u-w)^{2}\right], \\
N\left(Q_{i}^{(3)}\right)(u, w)= & \sigma_{i s}^{2} \sqrt{n_{i} n_{s}}\left(\frac{m}{2 \pi k T}\right)^{1 / 2} \frac{1}{\|u-w\|} \\
& \times \exp \left[-\frac{m}{8 k T} \frac{\left(u^{2}-w^{2}\right)^{2}}{\|u-w\|^{2}}-\frac{m}{8 k T}(u-w)^{2}\right] .
\end{aligned}
$$

Note that, in the case of one component gas, expressions (44-46) reduce to those presented by Grad in paper [5], for the intermolecular potential of hard-sphere type.

Kernels of the linearized reactive operators. The procedure adopted to obtain the representation of the kernels of the reactive operators is similar to the one used for the elastic operators. The starting point is the decomposition of the operator $\widehat{\mathscr{L}}_{i}^{R}(\underline{\hat{h}})$ into several contributions, in the form

$$
\widehat{\mathscr{L}}_{i}^{R}(\underline{\hat{h}})=-v_{i}^{R}(u) \hat{h}_{i}(u)-\mathscr{R}_{i}^{(1)}(\underline{\hat{h}})+\mathscr{R}_{i}^{(2)}(\underline{\hat{h}})+\mathscr{R}_{i}^{(3)}(\underline{\hat{h}}),
$$

where, for $i=1,2,3,4$,

$$
\begin{aligned}
& v_{i}^{R}(u) \hat{h}_{i}(u)=\hat{h}_{i} \beta_{i j} \sigma_{i j}^{2} \int_{\mathbb{R}^{3}} \int_{\mathbb{S}_{+}^{2}} M_{j} \Theta\left(\left\langle\varepsilon, \xi_{i}\right\rangle-\Gamma_{i j}\right)\left\langle\varepsilon, \xi_{i}\right\rangle d \varepsilon d c_{j}, \\
& \mathscr{R}_{i}^{(1)}(\underline{\hat{h}})=\beta_{i j} \sigma_{i j}^{2} \int_{\mathbb{R}^{3}} \int_{\mathbb{S}_{+}^{2}} M_{i}^{1 / 2} M_{j} \hat{h_{j}} \Theta\left(\left\langle\varepsilon, \xi_{i}\right\rangle-\Gamma_{i j}\right)\left\langle\varepsilon, \xi_{i}\right\rangle d \varepsilon d c_{j}, \\
& \mathscr{R}_{i}^{(2)}(\underline{\hat{h}})=\beta_{i j} \sigma_{i j}^{2} \int_{\mathbb{R}^{3}} \int_{\mathbb{S}_{+}^{2}} M_{j}^{1 / 2} M_{l}^{* 1 / 2} \hat{h}_{k}^{*} \Theta\left(\left\langle\varepsilon, \xi_{i}\right\rangle-\Gamma_{i j}\right)\left\langle\varepsilon, \xi_{i}\right\rangle d \varepsilon d c_{j}, \\
& \mathscr{R}_{i}^{(3)}(\underline{\hat{h}})=\beta_{i j} \sigma_{i j}^{2} \int_{\mathbb{R}^{3}} \int_{\mathbb{S}_{+}^{2}} M_{j}^{1 / 2} M_{k}^{* 1 / 2} \hat{h_{l}^{*}} \Theta\left(\left\langle\varepsilon, \xi_{i}\right\rangle-\Gamma_{i j}\right)\left\langle\varepsilon, \xi_{i}\right\rangle d \varepsilon d c_{j} .
\end{aligned}
$$

Again, the multiplication operator $v_{i}^{R}$ defined in (47) can be identified as a mean reactive collision frequency. Concerning the integral operators (48-50), the full representation of their kernels, for the general case of arbitrary molecular masses, is omitted here due to space limitations. The reader is addressed to Refs. [1, 2] for the details. Here we present the particular case of equal molecular masses, and only present the kernels of the operators $\mathscr{R}_{i}^{(1)}(\underline{\hat{h}})$, for $i=1,2,3,4$, and $\mathscr{R}_{1}^{(2)}(\underline{\hat{h}}), \mathscr{R}_{1}^{(3)}(\underline{\hat{h}})$,

$$
\begin{aligned}
N\left(\mathscr{R}_{i}^{(1)}\right)(u, w)= & \frac{1}{2} \beta_{i j} \sigma_{i j}^{2} \sqrt{n_{i} n_{j}}\left(\frac{m}{2 \pi k T}\right)^{3 / 2} \frac{\|u-w\|^{2}-\Gamma_{i j}^{2}}{\|u-w\|} \\
& \times \exp \left(-\frac{m\left(u^{2}+w^{2}\right)}{4 k T}\right), \quad i=1,2,3,4,
\end{aligned}
$$




$$
\begin{aligned}
& N\left(\mathscr{R}_{1}^{(2)}\right)(u, w)=\frac{1}{2} \beta_{12} \sigma_{12}^{2} \sqrt{n_{2} n_{4}}\left(\frac{m}{2 \pi k T}\right)^{3 / 2} \\
& \times \frac{\|u-w\|^{2}}{\left(\|u-w\|+\frac{Q_{R}}{m\|u-w\|}\right)^{3}} \Theta\left(\|u-w\|+\frac{Q_{R}}{m\|u-w\|}-\Gamma_{12}\right) \\
& \times\left[\frac{2 Q_{R}}{m\left(\|u-w\|+\frac{Q_{R}}{m\|u-w\|}\right)^{2} \sqrt{1-\frac{4 Q_{R}}{m\left(\|u-w\|+\frac{Q_{R}}{m \mid u-w \|}\right)^{2}}}}-\frac{\|u-w\|}{\|u-w\|+\frac{Q_{R}}{m\|u-w\|}}\right] \\
& \times \int_{L \perp(w-u)} \exp \left\{-\frac{m}{4 k T}\left[\left(u-L+\frac{2(w-u)}{1-\sqrt{1-\frac{4 Q_{R}}{m\left(\|u-w\|+\frac{Q_{R}}{m|u-w|}\right)^{2}}}}\right)^{2}\right.\right. \\
& \left.+\left(2 u-L-w+\frac{2(w-u)}{1-\sqrt{1-\frac{4 Q_{R}}{m\left(\|u-w\|+\frac{Q_{R}}{m \mid u-w \|}\right)^{2}}}}\right)^{2}\right] d L, \\
& N\left(\mathscr{R}_{1}^{(3)}\right)(u, w)=\beta_{12} \sigma_{12}^{2} \sqrt{n_{2} n_{3}}\left(\frac{m}{2 \pi k T}\right)^{3 / 2} \int_{D_{L}} \exp \left\{-\frac{m}{4 k T}[\right. \\
& \left.\left.\times\left(u+\frac{1}{2}\left(-1+\sqrt{1-\frac{4 Q_{R}}{m L^{2}}}\right) L\right)^{2}+\left(w+\frac{1}{2}\left(-1+\sqrt{1-\frac{4 Q_{R}}{m L^{2}}}\right) L\right)^{2}\right]\right\} \\
& \times \Theta\left(\|L\|-\Gamma_{12}\right) \frac{1}{\left\|w-u-\frac{1}{2}\left(1+\sqrt{1-\frac{4 Q_{R}}{m L^{2}}}\right) L\right\|} d L,
\end{aligned}
$$

where the integration domain $D_{L}$ is defined by

$$
D_{L}=\left\{L \in \mathbb{R}^{3}:\langle L, v-w\rangle=\frac{1}{2}\left(1+\sqrt{1-\frac{4 Q_{R}}{m L^{2}}}\right) L^{2}\right\} .
$$

\section{Final remarks}

The content of this paper is the first part of a work in progress on the SRS model for a quaternary mixture with no restriction on the molecular masses of the constituents. The properties of the linearized SRS system presented here, and especially the explicit representations of its kernels, are essential in obtaining detailed spectral analysis of the system. This in turn provides the asymptotic behavior of its evolution operator that is used in existence and stability of close to equilibrium solutions for 
the SRS system and the rigorous treatment of the hydrodynamical limits at the Euler and Navier-Stokes level.

Acknowledgements This research was partially supported by the FCT Phd Grant SFRH/BD/28795/2006, by the Research Centre of Mathematics of the University of Minho through the FEDER Funds "Programa Operacional Factores de Competitividade COMPETE", and by the Portuguese Funds through FCT - "Fundação para a Ciência e Tecnologia" within the Project PEst-C/MAT/UI0013/2011.

\section{References}

1. Carvalho, F., Mathematical methods for the Boltzmann equation in the context of chemically reactive gases. Doctoral Thesis, 2012, http://hdl. handle. net/1822/24430 Repositorium of the University of Minho.

2. Carvalho, F., Polewczak, J. and Soares, A. J., Representation of the kernels of the Simple Reacting Spheres system (2013). Computations available at http://w3.math.uminho.pt/ ajsoares/Kernels_SRS_Model

3. Cercignani, C., Theory and Application of the Boltzmann Equation. Scottish Academic Press, Edinburgh (1975)

4. Grad, H., Asymptotic Theory of the Boltzmann Equation II. In Rarefied Gas Dynamics 1 (Paris, 1962) Edited by J. A. Laurmann. New York: Academic Press, 26-59 (1963)

5. Grad, H., Asymptotic Theory of the Boltzmann Equation. Phys. Fluids 6, 147-181 (1963)

6. Kremer, G., Introduction to the Boltzmann Equation and Transport Processes in Gases. Springer, Berlin (2010)

7. Kremer, G., Oliveira F. and Soares, A. J. $\mathscr{H}$-Theorem and Trend to Equilibrium of Chemically Reactive Mixture of Gases. Kinetic and Related Models 2, 333-343 (2009)

8. Marron, M. T., Simple Collision Theory of Reactive Hard Spheres. J. Chem. Phys. 52, 4060$4061(1970)$

9. Moreau, M.: Formal study of a chemical reaction by Grad expansion of the Boltzmann equation. Physica A 79, 18-51 (1975)

10. Polewczak, J., The Kinetic Theory of Simple Reacting Spheres: I. Global Existence Result in a Dilute-Gas Case. J. Stat. Physics 100, 327-362 (2000)

11. Polewczak, J. and Soares, A. J., Kinetic Theory of Simple Reacting Spheres I, 27th International Symposium on Rarefied Gas Dynamics, 2010, AIP Conf. Proc. 1333, 117-122 (2011)

12. Present, R. D., On the velocity distribution in a chemically reacting gas. J. Chem. Phys. 31, 747-797 (1959)

13. Prigogine, I. and Xhrouet, E., On the perturbation of Maxwell distribution function by chemical reaction in gases. Physica XV, 913-932 (1949)

14. Ross, J. and Mazur, P., Some deductions from a formal statistical mechanical theory of chemical kinetics. J. Chem. Phys. 35, 19-28 (1961)

15. Shizgal, B. and Karplus, M., Nonequilibrium contributions to the Rate of Reaction. I. Perturbation of the Velocity Distribution Function. J. Chem. Phys. 52, 4262-4278 (1970)

16. Villani, C., A Review of Mathematical Topics in Collisional Kinetic Theory. Handbook of Mathematical Fluid Dynamics 1 (2002)

17. Xystris, N. and Dahler, J. S., Mass and momentum transport in dilute reacting gases. J. Chem. Phys. 68, 354-373 (1978)

18. Xystris, N. and Dahler, J. S., Kinetic Theory of Simple Reactive Spheres. J. Chem. Phys. 68, 387-401 (1978) 